\title{
Intrapartum maternal anaphylaxis to tramadol: a case report
}

\author{
Namkha Dorji ${ }^{1}$, Sangay Tshering ${ }^{2}$, and Tabassum Parveen ${ }^{3}$ \\ ${ }^{1}$ Jigme Dorji Wangchuck National Referral Hospital \\ ${ }^{2}$ JDWNR Hospital \\ ${ }^{3}$ Bangabandhu Sheikh Mujib Medical University
}

March 30, 2021

\begin{abstract}
Background: Tramadol causing intrapartum maternal anaphylaxis is unknown. Case: A 29-year-old, primigravida at $39+4$ weeks pregnancy had an anaphylactic reaction to injection tramadol. She was intubated, caesarean section done and health baby and mother were discharged. Conclusion: Staffs have to be aware of this uncommon anaphylactic reaction to tramadol.
\end{abstract}

\section{Introduction}

Anaphylaxis is a rapid onset, systemic allergic reaction involving activation of mast cells and/or basophils which is potentially life threatening (1). It is typically triggered by insects bite, rubber latex, foods and drugs through IgE activation (1). The estimated life time prevalence of anaphylaxis is between $0.05 \%$ and $2.0 \%$ (2). The prevalence of maternal anaphylaxis was 2.7 cases per 1,00,000 deliveries (3). However, there is no literature on tramadol causing anaphylaxis in pregnancy.

Tramadol is a centrally acting opioid analgesics that is commonly used for mild to moderate pain relief. Tramadol is in step 2 WHO step ladder of analgesic used for mild to moderate pain (4).

Tramadol has modest $\mu$ opioid receptors affinity and no affinity for $\delta$ or $x$ opioid receptors. The affinity of tramadol for $\mu$ receptors is 6000 -fold less than that of morphine. The metabolite M1 has 300 -fold higher affinity than tramadol but it is still much lower than that of morphine. Tramadol also inhibits neuronal reuptake of norephinephrine and serotonin (5). It is commonly used as labour analgesics in Bhutan.

Allergic reactions to tramadol whether anaphylaxis or $\operatorname{IgE}$ mediated is rare, and considered safe for use (5). There are few case reports of tramadol induced anaphylaxis in non-pregnant patients $(6,7)$. Therefore, this is a first report on tramadol induced intrapartum maternal respiratory arrest while the nation was in lockdown due to community transmission of SARS-CoV-19 from Bhutan. In this case, diagnosis of maternal collapse due to IV tramadol use is made on the basis of time between the drug administration and the patient's reaction.

\section{Case}

A 29-year-old primigravida at $39^{+4}$ weeks pregnancy was admitted to the emergency room (ER), Jigme Dorji Wangchuck National Referral hospital, Thimphu, Bhutan with early labour pain at 4 am on $31^{\text {st }}$ December, 2020. She had uneventful antenatal care. She didn't have any medical disorders and no known allergy to drugs, foods and insect bite. On admission, her vitals were stable. She was in early labour with cervical dilatation of $2 \mathrm{~cm}$.

While waiting for admission to maternity ward, her pain gradually increased in frequency, duration and intensity. For pain relief, emergency medical officer prescribed intramuscular tramadol 50mg initially. After 30 minutes, her pain score didn't improve. Immediately upon administration of Intravenous (IV) tramadol 
at 9:30 am, patient became drowsy with poor respiratory effort with SPO2 of $78 \%$ on room air, followed by absent spontaneous respiratory effort and loss of consciousness. There were no skin changes such as generalized hives or urticarial rashes. Bag and mask ventilation was initiated. Her peripheral pulses were palpable. Cardiac auscultation showed regular heart sounds. Injection Naloxone $0.4 \mathrm{mg}$ IV was given for two doses 30 seconds apart. Injection adrenaline $0.5 \mathrm{mg}$ IM and injection hydrocortisone $200 \mathrm{mg}$ IV were administered.

Since there was no return of spontaneous respiratory effort, respiratory arrest was diagnosed, and endotracheal intubation with $7.00 \mathrm{~mm}$ tube was done and connected to ventilator. She was positioned in left lateral position with 15 degree tilt. Cardiopulmonary resuscitation (CPR) with chest compression was not required as her cardiac function was stable. She was sedated with injection propofol $10 \mathrm{mg}$ IV and injection diazepam 10mg IV. Her SPO2 maintained at 100\%, blood pressure was 94/76 $\mathrm{mmHg}$, and pulse rate was 85 beats/minute. Fetal heart beat was 126 beats/minute. There is no cardiotocography facility in the ER for fetal heart beat tracing. Injection adrenaline was not repeated as the vitals were stable.

Patient was kept under ventilation as GeneXpert for SARS-COVID 19 report was pending. As soon as negative GeneXpert report was available, she was shifted to Operation Theatre. Emergency caesarean section was performed and a live female baby weighing $3.810 \mathrm{~kg}$ was delivered at $10.57 \mathrm{am}$. There was fresh meconium-stained amniotic fluid. APGAR score of was 8/10 and 10/10 at one and five minutes respectively.

Her vitals were stable during caesarean section. Extubation was done in the post-anesthesia care unit (PACU). She was then shifted to adult intensive care unit (AICU) and monitored closely. As her vitals were stable and SPO2 maintained with nasal oxygen at the flow rate of 2 litres/minute, she was shifted to maternity ward after 24 hours of close observation in AICU. She was weaned off the oxygen on the second day.

In the maternity ward, she made an uneventful recovery. Her anaphylaxis was uniphasic. A healthy mother and baby were discharged home on postoperative day 3. Her postnatal follow up were uneventful at one, three and six weeks.

\section{Discussion}

A revised definition for anaphylaxis is "a rapid onset serious systemic hypersensitivity reaction that has potential to cause death. It is characterized by compromise in breathing that is potentially life threatening and/or circulatory collapse, and may occur in the absence of typical skin features or circulatory shock" (8). Our patient developed sudden onset respiratory depression immediately following administration of IV tramadol which is consistent with the world allergy organization's (WAO) amended diagnostic criteria for anaphylaxis (8).

The prevalence of anaphylaxis in pregnancy was 2.7 cases per 1,00,000 deliveries ( $95 \%$ confidence interval, 1.7-4.2 cases per 1,00,000 deliveries). $\beta$-lactams antibiotics were responsible for anaphylaxis in 11out of 19 patients (3). Tramadol is commonly used as labor analgesics at Jigme Dorji Wangchuck National Referral Hospital, Bhutan. This is the first anaphylaxis during intrapartum period due to tramadol in this hospital. Whereas, the reported trigger for anaphylaxis during pregnancy was $\beta$-lactam antibiotics (3).

Ideally, perimortem caesarean section should be performed within 5 minutes of maternal cardiac arrest(9). However, in a systematic review on management of cardiac arrest in pregnancy, perimortem caesarean section within recommended 4-5 mins of cardiac arrest was performed in 8 out of 38 cases. Perimortem caesarean section in 30-38 weeks pregnancy done after recommended 5 minutes has shown successful maternal and neonatal outcomes (9). In our case, emergency caesarean section was done 87 minutes after maternal respiratory arrest and endotracheal intubation with live baby with APGAR score of 8/10 and 10/10 at one and five minutes of birth respectively. The successful neonatal outcome can be attributed to abrupt and successful intubation and maintenance of oxygen saturation. Her cardiac function was stable and she was kept in left lateral tilt of $15^{0}$ from the horizontal as per the International Liaison Committee on Resuscitation (ILCOR) consensus on science and treatment recommendations of 2005 (10) and the 2005 
European Resuscitation Council Guidelines (11).

The 57 minutes delay between onset of respiratory arrest and caesarean section was because of delay in GeneXpert report. As Bhutan was in second nationwide lockdown due to surge in community spread of SARS-COVID 19, emergency patients need COVID-19 GeneXpert report negative to shift from ER to operation room (OR) and there is no emergency OR available in ER.

The sensitivity of Xpert Xpress SARS-CoV-2 assays (GeneXpert) was $100 \%$ and the specificity was $80 \%$ for the diagnosis of COVID-19 in low middle income countries (LMICs) (12).

Healthy mother and newborn was discharged home on third postoperative day which corresponds with the length of hospital stay in other studies (3).

\section{Conclusion}

Tramadol causing respiratory depression in pregnancy should be treated in line of anaphylaxis. Prompt and effective resuscitation, keeping in left lateral position, and immediate delivery of fetus improves maternal and neonatal outcome.

\section{Patient Perspectives}

A healthy mother and neonate were discharged home. Family members were extremely grateful for saving mother and baby's life.

\section{Informed Consent}

The signed informed consent of patient is available

Fund : none

Conflict of interest: none

\section{References}

1. Simons FER, Frew AJ, Ansotegui IJ, Bochner BS, Golden DBK, Finkelman FD, et al. Risk assessment in anaphylaxis: Current and future approaches. J Allergy Clin Immunol. 2007;120(1 SUPPL.):2-24.

2. Lieberman P, Camargo CA, Bohlke K, Jick H, Miller RL, Sheikh A, et al. Epidemiology of anaphylaxis: Findings of the American College of Allergy, Asthma and Immunology Epidemiology of Anaphylaxis Working Group. Ann Allergy, Asthma Immunol [Internet]. 2006;97(5):596-602. Available from: http://dx.doi.org/10.1016/S1081-1206(10)61086-1

3. Mulla ZD, Ebrahim MS, Gonzalez JL. Anaphylaxis in the obstetric patient: Analysis of a statewide hospital discharge database. Ann Allergy, Asthma Immunol [Internet]. 2010;104(1):55-9. Available from: http://dx.doi.org/10.1016/j.anai.2009.11.005

4. Yang J, Bauer BA, Wahner-Roedler DL, Chon TY, Xiao L. The modified WHO analgesic ladder: Is it appropriate for chronic non-cancer pain? J Pain Res. 2020;13:411-7.

5. Grond S SA. Clinical pharmacology. Clin Pharmacokinet. 2004;43(13):879-923.

6. Nasarkhan, T. Das, I. Shastry VGR. A case of tramadol induced anaphylactic shock. Int J Curr Res. 2019;11(12):8950-3.

7. Mori F, Barni S, Manfredi M, Sarti L, Pecorari L, Pucci N, et al. Anaphylaxis to Intravenous Tramadol in a Child. Pharmacology. 2015;96(5-6):256-8.

8. Turner PJ, Worm M, Ansotegui IJ, El-Gamal Y, Rivas MF, Fineman S, et al. Time to revisit the definition and clinical criteria for anaphylaxis? World Allergy Organ J [Internet]. 2019;12(10):100066. Available from: https://doi.org/10.1016/j.waojou.2019.100066 
9. Jeejeebhoy FM, Zelop CM, Windrim R, Carvalho JCA, Dorian P, Morrison LJ. Management of cardiac arrest in pregnancy: A systematic review. Resuscitation [Internet]. 2011;82(7):801-9. Available from: http://dx.doi.org/10.1016/j.resuscitation.2011.01.028

10. Council ER. Part 2: Adult basic life support. Resuscitation. 2005;67(2-3):187-201.

11. Soar J, Deakin CD, Nolan JP, Abbas G, Alfonzo A, Handley AJ, et al. European Resuscitation Council Guidelines for Resuscitation 2005: Section 7. Cardiac arrest in special circumstances. Resuscitation. 2005;67(SUPPL. 1).

12. Rakotosamimanana N, Randrianirina F, Randremanana R, Raherison MS, Rasolofo V, Solofomalala GD, et al. GeneXpert for the diagnosis of COVID-19 in LMICs. Lancet Glob Heal. 2020;8(12):e1457-8. 